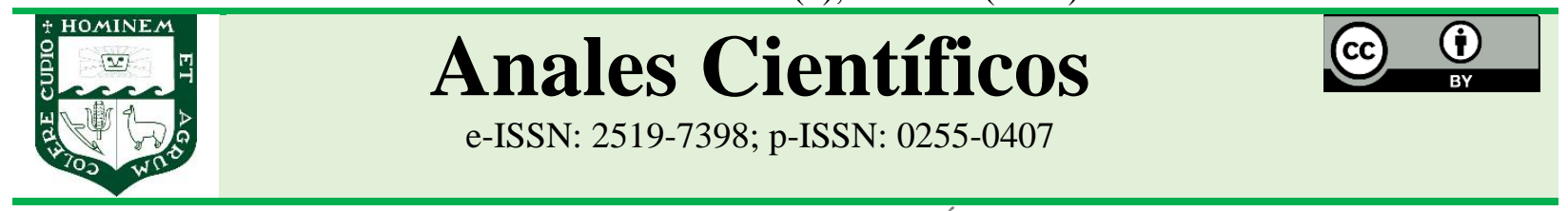

ARTÍCULO ORIGINAL - RESEARCH ARTICLE

http://dx.doi.org/10.21704/ac.v82i2.1763

\title{
IMPORTANCIA DE LA CARACTERIZACIÓN QUÍMICA DEL SUELO DE LAS ÁREAS VERDES MEDIANTE ESTADÍSTICO UNIVARIANTE Y ANÁLISIS DE CORRELACIÓN DE SPEARMAN EN LA UNIVERSIDAD PERUANA UNIÓN
}

\section{Importance of soil chemical characterization of green areas using univariate statistics and Spearman correlation analysis at the Universidad Peruana Unión}

\author{
Jhon Quispecuro-Huaman 1*(D); Diana Angel Rivas 1(D); Milda Cruz-Huaranga ${ }^{1}$ (D) \\ ${ }^{1}$ Facultad de Ingeniería y Arquitectura, Universidad Peruana Unión, Chosica, Perú \\ * E-mail: jhonquispecuro@upeu.edu.pe
}

Recibido: 16/03/2021; Aceptado: 16/10/2021; Publicado: 31/12/2021

\begin{abstract}
The main purpose of the study was to chemically characterize the green areas of the Universidad Peruana Unión to determine the influence of the different types of water on the dynamics between receiving soil, biological support and chemical inputs. The parameters analyzed, compared and correlated from the extraction of soil samples were $\mathrm{pH}$, Total Nitrogen, Organic Matter and Available Phosphorus. The univariate analysis of variance and Spearman's correlation coefficient were then used. The $\mathrm{pH}$ was the only significant parameter among the study areas, and high correlations were shown between Total Nitrogen, Organic Matter and Available Phosphorus. The type of irrigation water influenced the dynamic behavior of the green areas. Therefore, the study highlights to choose assertively the type of irrigation water, to manage both economic and sustainable water resources available (river water, groundwater and previously treated river water), to formulate, predict and accurately observe soil chemical dynamics and to evaluate extensive green areas of wild, forest, rural or agricultural nature in the immediate environment or integrated in the urban space according to the type of water used for irrigation.
\end{abstract}

Keywords. green areas | available phosphorus | organic matter | $\mathrm{pH} \mid$ nitrogen.

\section{RESUMEN}

El propósito principal del estudio fue caracterizar químicamente las áreas verdes de la Universidad Peruana Unión para determinar la influencia de los distintos tipos de agua en la dinámica entre suelo receptor, soporte biológico y aportes químicos. Los parámetros analizados, comparados y correlacionados a partir de la extracción de muestras de suelo fueron pH, Nitrógeno Total, Materia Orgánica y Fósforo disponible. Seguidamente se utilizó el estadístico análisis de varianza univariante y el coeficiente de correlación de Spearman. El pH resultó ser el único parámetro significativo entre las áreas de estudio, se mostraron correlaciones altas entre Nitrógeno Total, Materia Orgánica y Fósforo Disponible. El tipo de agua para riego influencio en el comportamiento dinámico de las áreas verdes. Por consiguiente, el estudio resalta elegir de manera asertiva el tipo de agua para riego, gestionar los recursos hídricos tanto económicos y sostenibles con los que se cuenta (agua del rio, agua subterránea y agua de rio previamente tratada), formular, predecir y observar con precisión la dinámica química del suelo y evaluar zonas verdes extensas 
de naturaleza silvestre, forestal, rural o agrícola en el entorno inmediato o integrados en el espacio urbano de acuerdo al tipo de agua utilizada para riego.

Palabras clave: áreas verdes | fósforo disponible | materia orgánica | pH | nitrógeno.

Forma de citar el artículo (Formato APA):

Quispecuro-Huaman, J., Angel, D., \& Cruz-Huaranga, M. (2021). Importancia de la caracterización química del suelo de las áreas verdes mediante estadístico univariante y análisis de correlación de Spearman en la Universidad Peruana Unión. Anales Científicos. 81(2), 09-18. http://dx.doi.org/10.21704/ac.v82i2.1763

Autor de correspondencia (*): Jhon Quispecuro Huaman. Email: jhonquispecuro@upeu.edu.pe

(C) Los autores. Publicado por la Universidad Nacional Agraria La Molina.

This is an open access article under the CC BY

\section{INTRODUCCIÓN}

El crecimiento cultural se ha visto influenciado en los dos últimos milenios principalmente por las condiciones hidromórficas e hidroclimatológicas (Toonen et al., 2021). Metodologías a nivel global se han ido desarrollando para evaluar la calidad del suelo, la gestión sostenible del agua, la incertidumbre climática (Hassani et al., 2020) y evaluar los efectos del cambio climático y la gestión de los recursos naturales en la calidad del suelo (Liu et al., 2020). Asimismo, el último informe sobre los riesgos mundiales (World Economic Forum, 2020) muestra por primera vez, los cinco primeros riesgos mundiales en términos de probabilidad (riesgos medioambientales) siendo uno de ellos la grave pérdida de biodiversidad, y el colapso de los ecosistemas (tierra y agua). El agua dulce es un recurso precioso, y su escasez puede provocar estrés hídrico, lo que repercute en la agricultura, la industria y otros sectores (Dingemans et al., 2020).

Akroush et al. (2016) indica que la tenencia de grandes extensiones de tierra conlleva a buscar tecnologías de captación para los recursos hídricos (agua subterránea, agua de lluvia, agua de mar, aguas residuales tratadas e inclusive no tratadas). Donde la reutilización de las aguas residuales se considera cada vez más una oportunidad para satisfacer la demanda de agua dulce debido al acelerado crecimiento de la población, la contaminación causada por éstos en los cuerpos de agua y la distribución no equitativa del recurso hídrico, han obligado a reusar las aguas residuales principalmente en las actividades agrícolas (Silva et al., 2008).

Aunque el uso de aguas residuales en la agricultura pueden traer beneficios, su uso incontrolado, a menudo trae impactos negativos sobre la salud humana (FAO, 2013). Sin embargo, si se implementan buenas prácticas de manejo, estos efectos podrían ser reducidos (OPS \& OMS, 2012). Cruz et al. (2016) recomienda el reúso de aguas residuales tratadas mediante macrófitas flotantes para riego de áreas verdes. Asimismo, Valdes et al. (2019) plantea que el uso de aguas tratadas para riego, disminuyen la demanda del uso de agua dulce y la sostenibilidad de acuíferos.

Sin embargo, Guo et al. (2017), menciona que el riego con agua tratada afecta la composición química del suelo, principalmente la conductividad eléctrica, cantidad de nitrógeno y materia orgánica afectando así la composición microbiana del suelo (Chodak et al., 2015). La relación directa entre composición química del suelo, la composición microbiana y el agua que se usa para su riego, influye también en la biodiversidad presente (Singh et al., 2016). Además, Sánchez et al. (2019) justifica la importancia de identificar las propiedades químicas del suelo, ya que éstas definen el comportamiento dinámico (transporte, infiltración, adsorción, absorción y liberación gaseosa) de compuestos químicos del agua. De Oliveira et al. (2017) indica que el uso de aguas residuales proporciona incrementos a las concentraciones químicas originales de las áreas regadas (Simonete et al., 2003). El riego debe ser realizado teniendo en cuenta las características iniciales del suelo en armonía con las características del agua (Sánchez et al. 2019).

Zamora et al. (2008) manifestó la importancia de evaluar los efectos del riego en áreas verdes con aguas residuales, ya que se han evaluado cambios positivos y negativos en las propiedades químicas del suelo. Por tanto, es necesario evaluar el uso del agua tratada para riego de áreas verdes, cuantificando su impacto en las propiedades químicas del suelo para asegurar que su uso no solo mejora la fertilidad y productividad del suelo, sino que también, mantiene la calidad de los 
recursos y asegura que no causen problemas ambientales o de salud pública.

Es por ello, que este trabajo de investigación tuvo por objetivo caracterizar las propiedades químicas $(\mathrm{pH}, \%$ materia orgánica, fósforo disponible y nitrógeno total) del suelo de las áreas verdes mediante estadístico univariante y análisis de correlación de Spearman, que podría ser utilizado para resaltar la influencia de los tipos de agua utilizados para el riego, mejorando la gestión del recurso hídrico disponibles de acuerdo a las necesidades específicas del riego de las áreas verdes en armonía con la dinámica entre suelo receptor, soporte biológico y aportes químicos

\section{MATERIALES Y MÉTODOS}

\section{Área de estudio}

El área de estudio se encuentra ubicado en el campus de la Universidad Peruana Unión (1159'30,73"S, $76^{\circ} 50$ '24,56"O), distrito de Lurigancho Chosica - Lima (Figura 1). La altitud del área de estudio oscilo entre
516 y $521 \mathrm{msnm}$. Esta área presentó un clima húmedo debido a la influencia de la corriente marina de Humboldt, una temperatura y precipitación media anual de $19,2{ }^{\circ} \mathrm{C}$ y $2 \mathrm{ml}$ respectivamente y la velocidad del viento promedio anual es de $96,5 \mathrm{~km} / \mathrm{d}$.

El tipo de suelo principal en el área de estudio posee afloramientos rocosos, estratos de grava que conforman los conos de deyección de los ríos Rímac y Chillón y los estratos de grava coluvial-eluvial de los pies de la ladera (SINIA, 2012).

El área de estudio fue dividida en tres áreas principales de acuerdo con la información obtenida por Gerencia de Servicios de la Universidad Peruana Unión: área 1 "área de campamento" que es regado con agua residual proveniente del canal (Río Rímac), área 2 "área de los huarangos" que es regado con agua residual tratada mediante macrófitas flotantes proveniente de la laguna La Mansión y área 3 "área de las residencias" que es regado con agua subterránea.

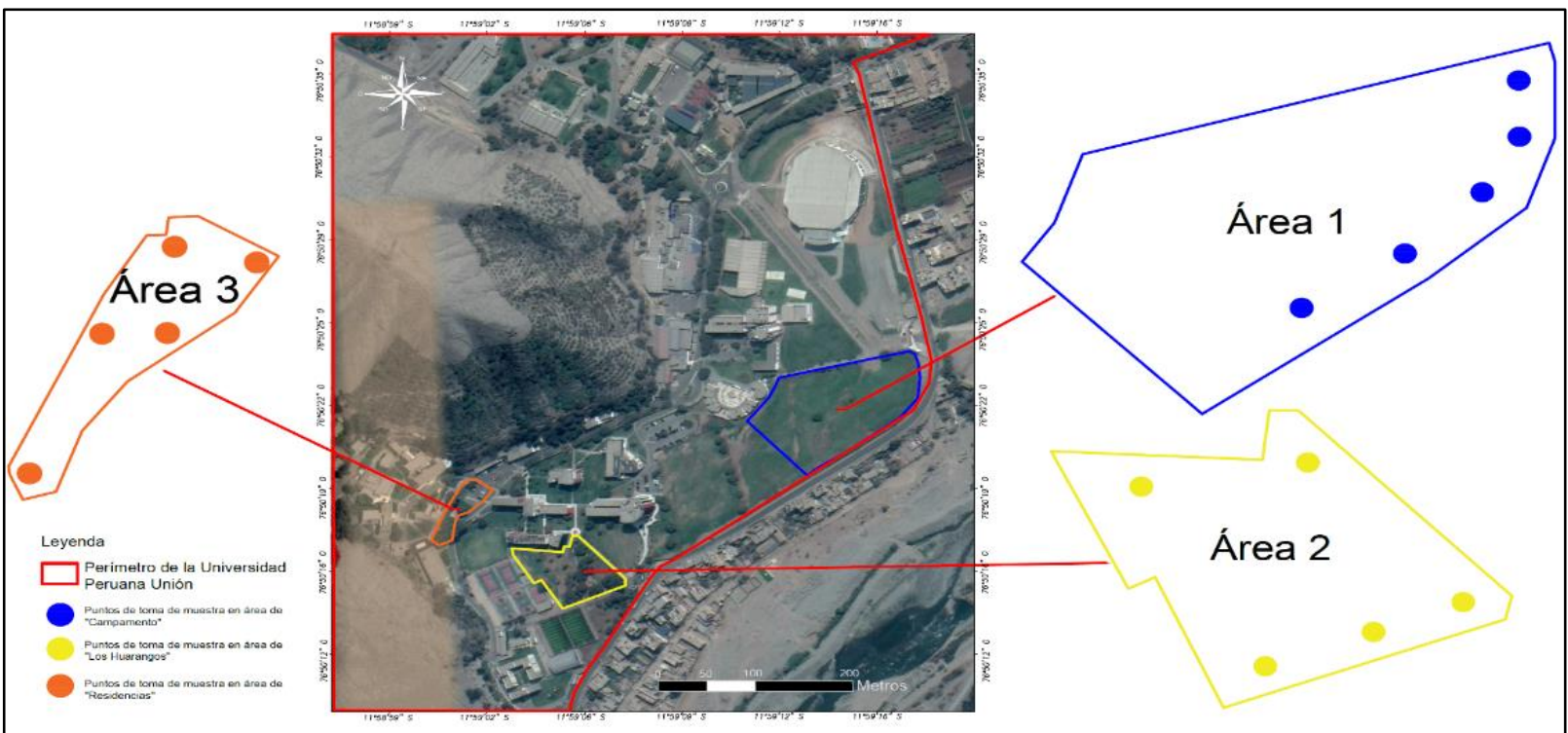

Figura 1. Universidad Peruana Unión. Punto de muestreo distribuido en diferentes áreas de acuerdo con la calidad del agua empleada para su riego.

\section{Obtención de muestras y análisis}

Los lugares de muestreo se observan en la Figura 1. Las muestras fueron recopiladas en enero del 2021 de las tres áreas de estudio, recopilando cinco muestras por cada área, para este fin se realizaron calicatas de 30 x $30 \mathrm{~cm}$ con una profundidad de $50 \mathrm{~cm}$ y se extrajo suelo para la muestra, posterior a ello, se tamizó y pesó $1 \mathrm{~kg}$ por muestra, luego fueron almacenados en bolsas ziploc con respectiva codificación (MINAM, 2014). 
Los análisis químicos $(\mathrm{pH}, \%$ de materia orgánica, fósforo disponible y nitrógeno total) fueron realizados en el Laboratorio de Análisis de Suelos, Plantas, Aguas y Fertilizantes (LASPAF) de la Universidad Nacional Agraria La Molina (UNALM). Para ello, las muestras se secaron a temperatura ambiente $\left(26{ }^{\circ} \mathrm{C}\right)$ y bajo sombra, se molieron y tamizaron con un tamiz ( $2 \mathrm{~mm}$ y $0,5 \mathrm{~mm}$ de diámetro), para los análisis de $\mathrm{pH}$ (medición en el potenciómetro de la suspensión de suelo: agua en relación 1:1), para materia orgánica (método de Walkley y Black, oxidación del carbono orgánico con dicromato de potasio), también para nitrógeno total (método de Kjeldahl) y para Fósforo disponible (método de Olsen modificado, extracción con $\mathrm{NaHCO}_{3}$ 0,5 N; pH 8,5) (LASPAF, 2021).

\section{Análisis estadístico}

Las características químicas de los distintos lugares de muestreo en las áreas 1; 2 y 3 presentaron una distribución normal, se utilizó el Análisis de Varianza (ANOVA) para identificar la diferencia significativa entre las medias de tres o más grupos (Lawson, 2015),esta prueba nos permitió valorar el efecto de la variable independiente sobre las variables dependientes con un nivel de significancia de 0,05 . Se utilizó el análisis de correlación de Spearman para examinar las relaciones entre las características químicas (Zhou \& Li, 2020) utilizando el software de acceso libre R estudio (RStudio Team, 2020).

\section{RESULTADOS Y DISCUSIÓN}

\section{Características químicas de las áreas de estudio}

En la Tabla 1, se presenta los niveles de pH, Nitrógeno Total, Materia Orgánica y Fósforo Disponible de áreas regadas con agua del canal $(1, n=5)$, con agua de la laguna $(2, n=5)$ y con agua subterránea $(3, n=5)$. De la Tabla 1, se encontró un $\mathrm{pH}$ (de las tres áreas estudiadas) de 7,88 máximo, mínimo de 7,23 y una media de 7,53 .

Tabla 1. Resultados de los análisis realizados en el laboratorio (LASFAP).

\begin{tabular}{|c|c|c|c|c|}
\hline \multirow{2}{*}{ Area } & pH & Nitrógeno Total & Materia Orgánica & Fósforo Disponible \\
\hline & $(1: 1)$ & $\%$ & $\%$ & ppm \\
\hline \multirow{5}{*}{1} & 7,60 & 0,11 & 1,68 & 21,0 \\
\hline & 7,48 & 0,10 & 1,35 & 20,5 \\
\hline & 7,23 & 0,08 & 0,94 & 17,4 \\
\hline & 7,64 & 0,10 & 1,14 & 26,2 \\
\hline & 7,60 & 0,15 & 2,09 & 29,7 \\
\hline \multirow{5}{*}{2} & 7,60 & 0,12 & 1,41 & 18,4 \\
\hline & 7,31 & 0,13 & 1,61 & 14,2 \\
\hline & 7,43 & 0,10 & 1,14 & 23,1 \\
\hline & 7,52 & 0,10 & 1,35 & 16,1 \\
\hline & 7,27 & 0,15 & 2,29 & 22,7 \\
\hline \multirow{5}{*}{3} & 7,56 & 0,22 & 3,09 & 18,6 \\
\hline & 7,72 & 0,08 & 0,74 & 19,8 \\
\hline & 7,72 & 0,13 & 1,88 & 18,3 \\
\hline & 7,88 & 0,14 & 1,95 & 15,9 \\
\hline & 7,64 & 0,28 & 3,50 & 78,5 \\
\hline \multirow{3}{*}{ Resumen } & Min $: 7,23$ & Min :0,08 & Min :0,74 & Min $: 14,20$ \\
\hline & Mean :7,53 & Mean :0,13 & Mean $: 1,73$ & Mean :24,03 \\
\hline & $\operatorname{Max}: 7,88$ & Max $: 0,28$ & $\operatorname{Max}: 3,50$ & Max :78,50 \\
\hline
\end{tabular}

Para el Nitrógeno Total se encontró un máximo porcentaje de 0,28, mínimo de 0,08 y una media de 0,13. Para Materia Orgánica se encontró un máximo porcentaje de 3,5\%, mínimo de 0,74 y una media de 1,73 y para el Fósforo Disponible se encontró una concentración máxima de 78,50ppm, mínimo de 14,20 ppm y una media de 24,03 ppm. Los niveles de $\mathrm{pH}$ se encuentran en el rango observado del agua proveniente de la laguna "La Mansión", el agua del canal y agua subterránea en la Universidad Peruana Unión (Giraldo, 2018) y (Jurado et al., 2014). Para el Nitrógeno Total se encontró una mayor media en el área 3. De acuerdo con Su et al. (2020) el contenido del nitrógeno en el agua subterránea se incrementa por 
la presencia de aguas residuales(agua del canal y agua de la Laguna), el tipo de suelo explicaría los resultados obtenidos que se incrementó por la tasa de infiltración de aguas superficiales (SINIA, 2012). De igual forma para Materia Orgánica se observó que los resultados no varían significativamente ya que se mantuvo la misma profundidad para la extracción de las 15 muestras(Díaz Lezcano et al., 2020).

En caso del Fósforo Disponible, se presentó el mayor valor de la media en el área 3, esto de dio debido a la presencia de un resultado atípico, el cual representa en sí mismo más del $50 \%$ de la media para el total de resultados en al área 3, el valor atípico podría deberse a la presencia de fertilizantes utilizados por Gerencia de Servicios de la Universidad Peruana Unión para el mejoramiento estético de los lugares que están contemplados en el área 3(pequeños jardines)

\section{Comparaciones de propiedades químicas del suelo} En la Figura 2, se muestra el análisis de ANOVA de Welch y Tukey test para las 3 áreas de estudio. Para el $\mathrm{pH}$ (Figura 2) se muestra en el área 3 una media mayor de $\mathrm{pH}(\mu=7,70)$, comparadas con las áreas $1(\mu=7,45)$ y $2(\mu=7,46)$. Asimismo, es observado diferencia significativa $(\mathrm{p}<0,05)$ entre el área 3 y las áreas 2 y 1 , pero no fue encontrado diferencia significativa $(\mathrm{p}>$ 0,05) entre el área 1 y 2. Zamora et al. (2008) mencionan que las aguas residuales (área 1 y 2)contribuyen en la disminución de los niveles de $\mathrm{pH}$ en el suelo, esto explicaría por qué el pH en estas dos áreas es menor al área 3(regada con agua subterránea). Jurado et al. (2014) observo el pH $(7,8)$ del agua subterránea en la Universidad Peruana Unión. Por otro lado, Luna \& Aburto (2014) indican que el nivel de pH dentro del intervalo (6,5 a 8) no afectan negativamente en la composición química del suelo.

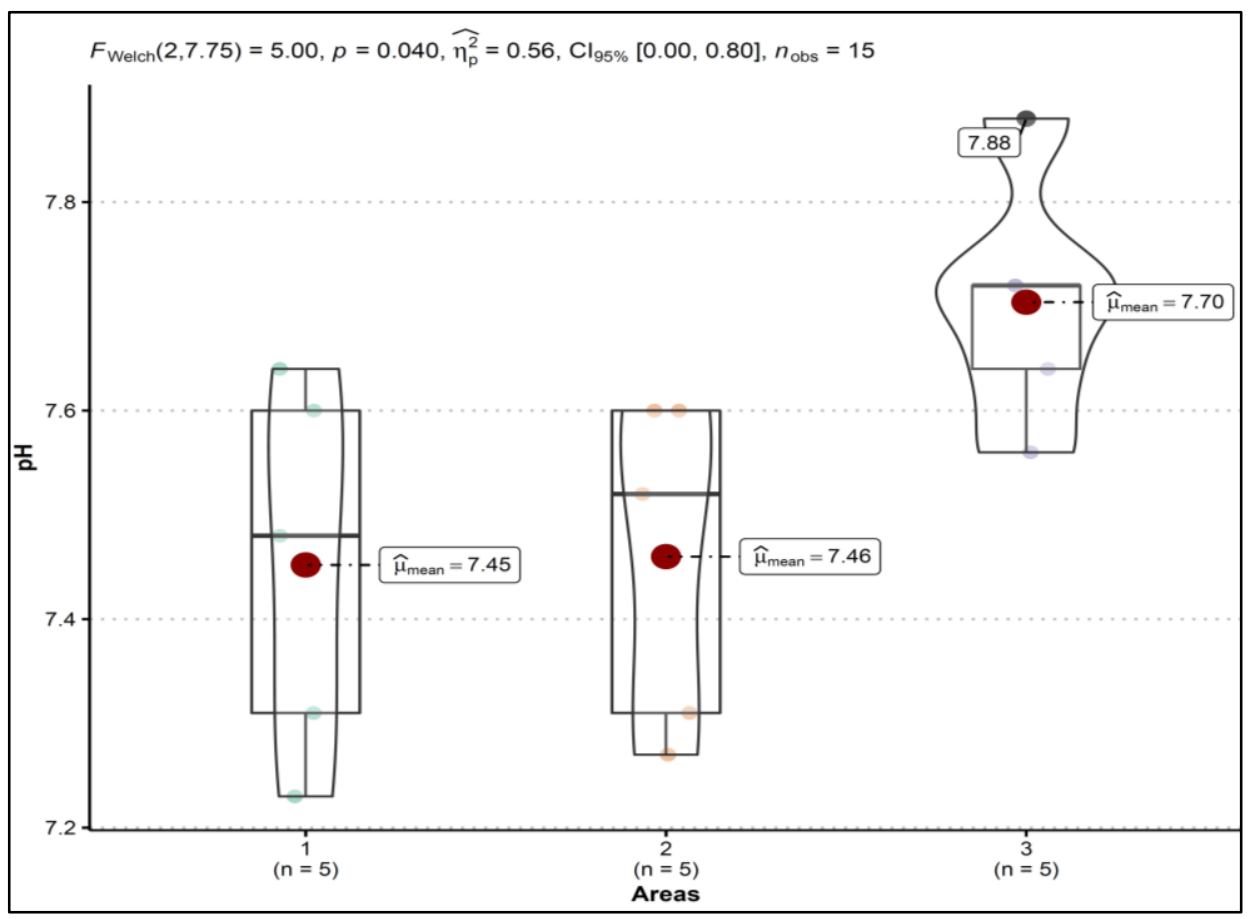

Figura 2. ANOVA de Welch para el análisis de varianza unidireccional para el pH. 


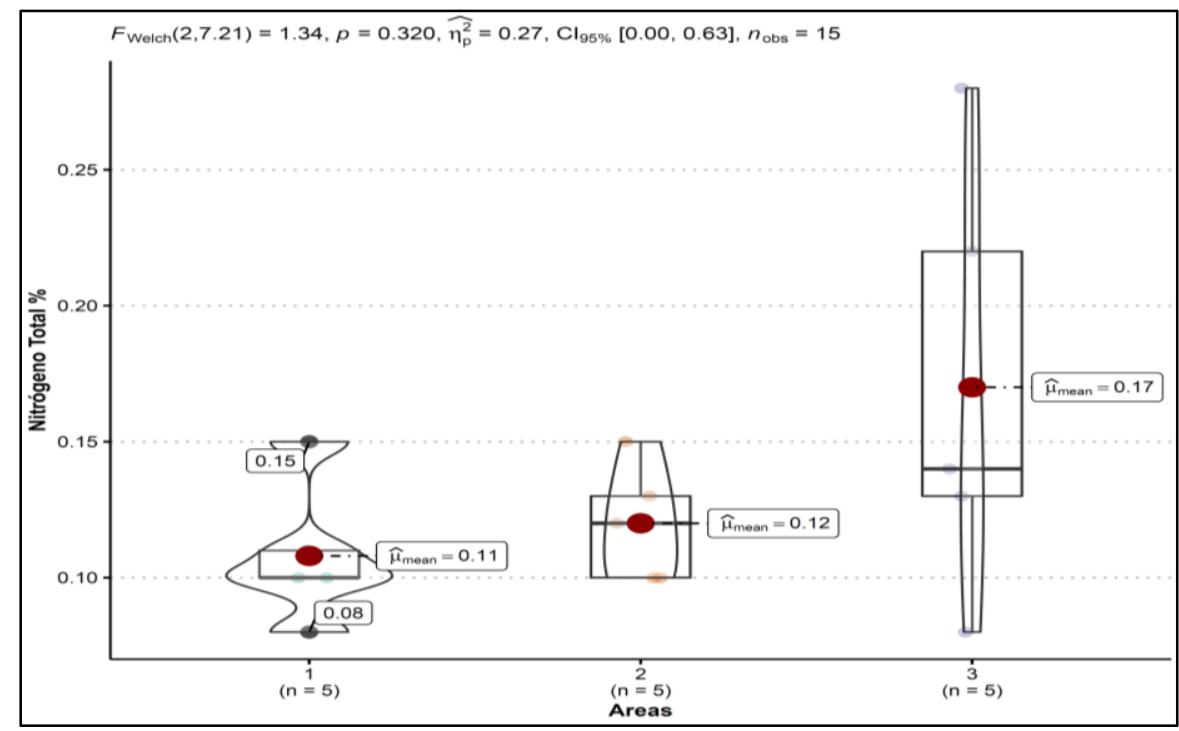

Figura 3. ANOVA de Welch para el análisis de varianza unidireccional para el Nitrógeno Total.

En la Figura 3, se muestra el análisis de ANOVA de Welch y Tukey test para las 3 áreas de estudio. Para el Nitrógeno Total (Figura 3) se muestra en el área 3 una media mayor de Nitrógeno Total $(\mu=17 \%)$, comparadas con las áreas $1(\mu=0,11 \%)$ y $2(\mu=0,12$ $\%)$. Asimismo, no se observó diferencia significativa $(\mathrm{p}<0,05)$ entre ninguna de las 3 áreas. Se muestra un patrón consecuente (repite el orden jerárquico de las medias) de acuerdo al porcentaje de materia Orgánica en las tres áreas de estudio. Asimismo, de acuerdo con Acevedo et al. (2011) al aumentar el porcentaje de materia orgánica en el suelo (1\% a $17 \%)$, incrementa el porcentaje de Nitrógeno Total en $40 \%$, esto se debería a la correlación positiva alta existente entre los dos parámetros $(0,973)$.

En la Figura 4, se muestra el análisis de ANOVA de Welch y Tukey test para las 3 áreas de estudio. Para la Materia Orgánica (Figura 4) se muestra en el área 3 una media mayor de Materia Orgánica $(\mu=2,23 \%)$, comparadas con las áreas $1(\mu=1,54 \%)$ y $2(\mu=1,44$ $\%)$. Asimismo, no se observó diferencia significativa $(\mathrm{p}<0,05)$ entre ninguna de las 3 áreas. La Materia Orgánica en el área 3 presenta una media con mayor valor y considerando el coeficiente de correlación alto con el Nitrógeno Total y el Fósforo Disponible (0,973 y 0,625) Bunkin et al. (2020) resaltan la presencia de la materia orgánica en suelos con interacciones biomoleculares (coeficiente de correlación alta y positiva) entre Fósforo Disponible y Nitrógeno Total.
Sin embargo Konrad \& Castilhos (2002) aluden que a largo plazo el agua residual tratada eficientemente y a mayor contacto con el suelo ayuda a aumentar la concentración de materia orgánica y nitrógeno total del mismo (Giraldo, 2018), lo cual sería beneficioso para las áreas 1 y 2.

En la Figura 5, se muestra el análisis de ANOVA de Welch y Tukey test para las 3 áreas de estudio. Para el Fósforo Disponible (Figura 5) se muestra en el área 3 una media mayor de Fósforo Disponible $(\mu=78,50$ ppm) comparadas con las áreas $1(\mu=22,96 \mathrm{ppm})$ y 2 $(\mu=18,90 \mathrm{ppm})$. Asimismo, no se observó diferencia significativa $(\mathrm{p}<0,05)$ entre ninguna de las 3 áreas. Por otro lado, el Fósforo Disponible representan entre el $27 \%$ y $68 \%$ de las concentraciones disponibles en el suelo y esta dinámica depende de prácticas de labranza y concentraciones de Fósforo no soluble en agua (Amery et al., 2021), si bien las áreas con concentraciones individuales de Fósforo Disponible se encuentran en las áreas 1 y 2 por la correlación con la Materia Orgánica y Nitrógeno Total (Acevedo et al., 2011). Sin embargo, un valor atípico como el que se muestra entre los resultados posiblemente se deba a prácticas continuas en jardines (área 3) y no se muestren en áreas más extensas de Grass (área 1) y arboles (área 2). 


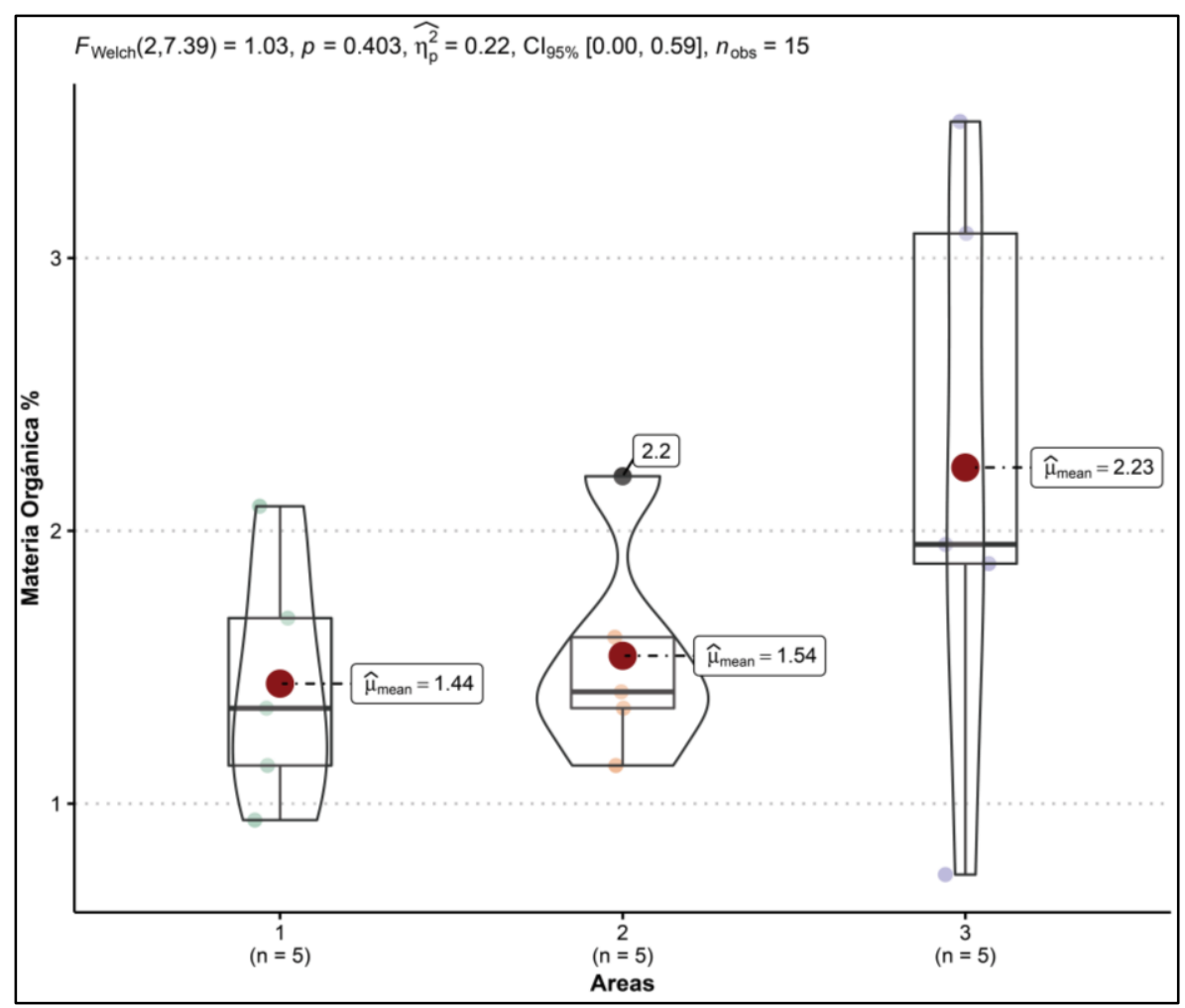

Figura 4. ANOVA de Welch para el análisis de varianza unidireccional para Materia Orgánica.

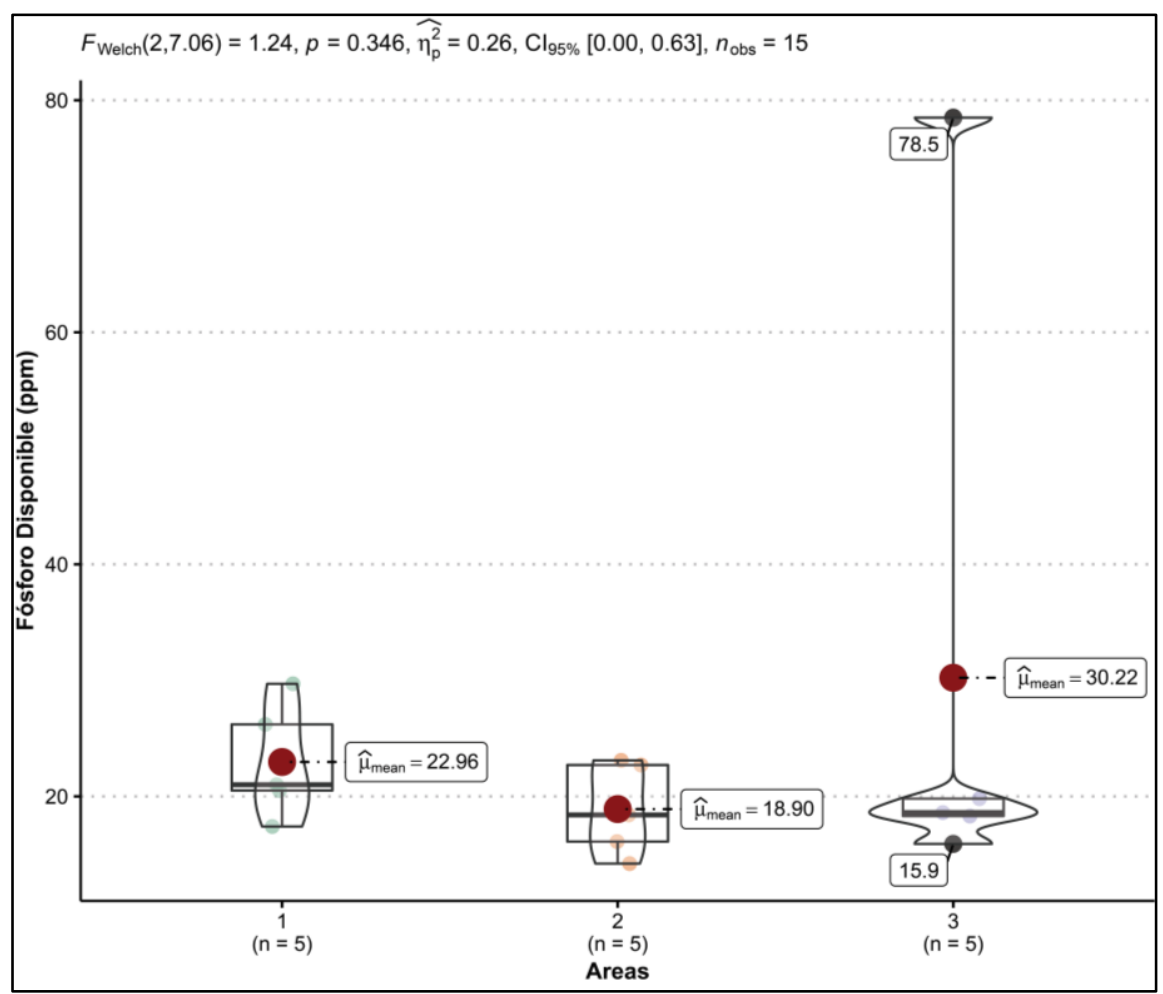

Figura 5. ANOVA de Welch para el análisis de varianza unidireccional para el Fósforo Disponible. 


\section{Correlación de Spearman entre propiedades químicas}

El análisis de correlación de Spearman mostró en la Tabla 2 coeficientes para las propiedades químicas del suelo. Entre el Nitrógeno Total y la Materia Orgánica existe una correlación alta $(0,973)$, seguido del Nitrógeno Total y Fósforo Disponible $(0,736)$, luego se encuentra la correlación entre Materia orgánica y Fósforo Disponible $(0,625)$, Ahora bien, las siguientes correlaciones tendieron a bajar para el nitrógeno Total y el $\mathrm{pH}(0,151)$, de igual forma, para la Materia Orgánica con el $\mathrm{pH}(0,121)$ y finalmente para el Fósforo Disponible y pH $(0,039)$. Cabe mencionar que no se encontró correlaciones negativas. Similares resultados para los coeficientes entre Materia Orgánica, Fósforo Disponible y Nitrógeno Total fueron mostrados por Acevedo et al.(2011), (Bunkin et al., 2020). Por otro lado aunque presentan una correlación positiva con el $\mathrm{pH}$, esta no es alta $(0,121$; 0,039 y 0,151 ). Luna \& Aburto (2014) indican el rango adecuado del $\mathrm{pH}$ como factor ambiental (Zhang \& Furman, 2021). La relación lineal es un factor importante para inferir las características químicas(evaluar patrones), independiente del tamaño de la muestra.(Zhang et al., 2016)

Tabla 2. Análisis de correlación entre variables

\begin{tabular}{lllll}
\hline & $\mathbf{p H}$ & $\begin{array}{l}\text { Materia } \\
\text { Orgánica }\end{array}$ & $\begin{array}{l}\text { Fósforo } \\
\text { Disponible }\end{array}$ & $\begin{array}{l}\text { Nitrógeno } \\
\text { Total }\end{array}$ \\
\hline $\mathrm{pH}$ & 1 & - & - & - \\
\hline $\begin{array}{l}\text { Materia } \\
\text { Orgánica }\end{array}$ & 0,121 & 1 & - & - \\
\hline $\begin{array}{l}\text { Fósforo } \\
\text { Disponible }\end{array}$ & 0,039 & 0,625 & 1 & - \\
\hline $\begin{array}{l}\text { Nitrógeno } \\
\text { Total }\end{array}$ & 0,151 & 0,973 & 0,736 & 1 \\
\hline
\end{tabular}

\section{CONCLUSIONES}

Se caracterizo químicamente el suelo de las áreas verdes y se demostró la influencia significativa del riego con los distintos tipos de agua mediante estadístico univariante y análisis de correlación de Spearman, demostrando la importancia en la elección del tipo de agua de acuerdo a necesidades específicas del caso y en armonía con los servicios ambientales que brindan las áreas verdes (regulación hídrica, captura de carbono, biodiversidad y recreación). Sin embargo, la gestión del recurso hídrico disponible presenta diferentes métodos de obtención ya sean mediante previo tratamiento (aguas tratadas), toma directa (agua del rio) o por succión (agua subterránea), cada una difiere en costo, tiempo de obtención y disponibilidad. Lo cual conocer si las características químicas del suelo influenciada por el tipo de agua es el ideal para el uso que se pretende dar conlleva al aprovechamiento del recurso hídrico de manera sostenible, eficiente y con menor impacto hacia el ambiente.

Agradecimiento

A la Universidad Peruana Unión por permitirnos realizar el proyecto dentro de sus instalaciones.

\section{Conflictos de intereses}

Los autores firmantes del presente trabajo de investigación declaran no tener ningún potencial conflicto de interés personal o económico con otras personas u organizaciones que puedan influir indebidamente con el presente manuscrito.

\section{Contribuciones de los autores}

Preparación y ejecución: JQH, DAR; Desarrollo de la metodología: JQH, DAR, MCH; Concepción y diseño: JQH, DAR, MCH; Edición del artículo: JQH, DAR, $\mathrm{MCH}$; Supervisión del estudio: JQH, DAR, MCH.

\section{REFERENCIAS}

- Acevedo, C., Sanchez, Á., Hernández, E., \& Ameéndola, R. (2011). Concentración de nitrógeno en suelo por efecto de manejo orgánico y convencional. Terra Latinoamericana, 29(3), 325332. https://www.redalyc.org/pdf/573/57321283011.pdf

- Akroush, S., Dehehibi, B., Dessalegn, B., AlHadidi, O., \& \& Abo-Roman, M. (2016). Factors Affecting the Adoption of Water Harvesting Technologies: A Case Study of Jordanian Arid Area. Sustainable Agriculture Research, 6(1),80. https://doi.org/10.5539/sar.v6n1p80

- Amery, F., Vandecasteele, B., D’Hose, T., Nawara, S., Elsen, A., Odeurs, W., Vandendriessche, H., Arlotti, D., McGrath, S. P., Cougnon, M., \& Smolders, E. (2021). Dynamics of soil phosphorus measured by ammonium lactate extraction as a function of the soil phosphorus balance and soil properties. Geoderma, 385. https://doi.org/10.1016/j.geoderma.2020.114855

- Bunkin, N. F., Glinushkin, A. P., Shkirin, A. V., Ignatenko, D. N., Chirikov, S. N., Savchenko, I. V., 
Meshalkin, V. P., Samarin, G. N., Maleki, A., \& Kalinitchenko, V. P. (2020). Identification of Organic Matter Dispersions Based on Light Scattering Matrices Focusing on Soil Organic Matter Management. ACS Omega, 5(51), 3321433224. https://doi.org/10.1021/acsomega.0c04906

- Chodak, M., Gołębiewski, M., MorawskaPłoskonka, J., Kuduk, K., Niklińska, M. (2015). Soil chemical properties affect the reaction of forest soil bacteria to drought and rewetting stress. Annals of Microbiology, 65(3), 1627-1637. https://doi.org/10.1007/s13213-014-1002-0

- Cruz, M., Carbo, N., Gonzales, J. L. L., Tito, G. M., Depaz, K., Torres, S., Núñez, R., Torres, J., \& Quispe, W. (2016). Tratamiento De Las Aguas De La Laguna "Mansión" Mediante La Especie Eichhorniacrassipes, Para El Riego De Áreas Verdes En La Universidad Peruana Unión. IOSR Journal of Agriculture and Veterinary Science, 09(08), 53-65. https://doi.org/10.9790/23800908025365

- De Oliveira, J. F., Rodrigues, F. N., Fia, R., Vilela, H. S., \& Landim, D. V. (2017). Chemical properties of soil fertirrigated with dairy and slaughterhouse wastewater. Engenharia Agricola, 37(6), 1244-1253

- Díaz Lezcano, M. I., Gamarra Lezcano, C. C., Ruiz Diaz, S., \& Vera de Ortiz, M. (2020). Contenido de materia orgánica en suelos de sistemas silvopastoriles establecidos en el Chaco Central paraguayo. Revista de La Sociedad Científica Del Paraguay, 25(2), 131-143. https://doi.org/10.32480/rscp.2020.25.2.131

- Dingemans, M., Smeets, P., Medema, G., Frijns, J., Raat, K., van Wezel, A., \& Bartholomeus, R. (2020). Responsible Water Reuse Needs an Interdisciplinary Approach to Balance Risks and Benefits. Water, 12(5), 1264. https://doi.org/10.3390/w12051264

- FAO. (2013). Reutilización del agua y agricultura: Beneficios para todos.

- Giraldo, C. (2018). Eficiencia de la especie Eichhornia Crassipes - Jacinto de Agua en el Tratamiento del Agua Residual de la Laguna "La Mansión" para el riego de las áreas verdes de la Universidad Peruana Unión, Período 2015 [Universidad Nacional Santiago Antúnez de Mayolo]. In Universidad Nacional Santiago
Antúnez de Mayolo. http://repositorio.unasam.edu.pe/handle/UNASA $\mathrm{M} / 4254$

- Guo, W., Andersen, M. N, Qi, X., Li, P., Li, Z., Fan, X., \& Zhou, Y. (2017). Effects of reclaimed water irrigation and nitrogen fertilization on the chemical properties and microbial community of soil. Journal of Integrative Agriculture, 16(3), 679-690. https://doi.org/10.1016/S20953119(16)61391-6

- Hassani, A., Azapagic, A., \& Shokri, N. (2020). Predicting long-term dynamics of soil salinity and sodicity on a global scale. Proceedings of the National Academy of Sciences, 117(52), 3301733027. https://doi.org/10.1073/pnas.2013771117

- Konrad, E., \& Castilhos, D. (2002). Alterações químicas do solo e crescimento do milho decorrentes da adição de lodos de curtume. Revista Brasileira de Ciência Do Solo, 26(1), 257-265. https://doi.org/10.1590/S010006832002000100027

- LASPAF. (2021). Metodologías empleadas en el Laboratorio de análisis de Suelos, Plantas, Aguas, y Fertilizantes de la Universidad Nacional Agraria La Molina. http://www.laspaf.com/assets/metodologia/analisi s-suelos.pdf

- Lawson, J. (2015). Design and Analysis of Experiments with $R$ CHAPMAN \& HALL/CRC Texts in Statistical Science Series Series Editors Practical Multivariate Analysis, Fifth Edition Interpreting Data: A First Course in Statistics Introduction to Probability with R K. Baclawski (F. Dominici, J. J. Faraway, M. Tanner, J. Zidek, F. Abramovich, Y. Ritov, A. Afifi, S. May, V. A. Clark, D. G. Altman, A. J. B. Anderson, S. Banerjee, A. Roy, C. R. Bilder, T. M. Loughin, D. Bissell, J. K. Blitzstein, \& J. Hwang (eds.)). https://doi.org/978-1-4987-2848-5

- Liu, Y., Zhang, J., \& Ge, Q. (2020). The optimization of wheat yield through adaptive crop management in a changing climate: evidence from China. Journal of the Science of Food and Agriculture, jsfa. 10993. https://doi.org/10.1002/jsfa.10993

- Luna, V., \& Aburto, S. (2014). Sistema de humedales artificiales para el control de la eutroficación del lago del Bosque de San Juan de 
Aragón | Elsevier Enhanced Reader. TIP Revista Especializada En Ciencias Químico-Biológicas, 1(17), $32-55$. https://reader.elsevier.com/reader/sd/pii/S1405888 $\mathrm{X} 14703183$ ?token $=90 \mathrm{C0A320188 \text {AD7D0EEBE0 }}$ A80EC4DA650BB08A3172905A4FAEC2618AD 956DF2E30FAB584A37B0CA032225B1AC0216 47BE

- MINAM. (2014). Guia para el muestreo de suelos (Primera ed).

- OPS, \& OMS. (2012). OPS/OMS Perú Tratamiento Adecuado de Aguas Residuales es el gran Reto ante Crecimiento Urbano. OMS. https://www.paho.org/per/index.php?option=com content $\&$ view $=$ article $\&$ id=1964:tratamientoadecuado-aguas-residuales-gran-reto-antecrecimiento-urbano\&Itemid $=900$

- RStudio Team. (2020). Integrated Development for $R$. RStudio. https://support.rstudio.com/hc/enus/articles/206212048-Citing-RStudio

- Sánchez, D., Navarro, C., Rentería, M., Sánchez, J., Herrera, E., \& Rose, J. (2019). Treated wastewater viability of use in green areas according to nitrogen compounds concentration. Water Practice and Technology, 14(2), 457-470. https://doi.org/10.2166/wpt.2019.035

- Silva, J., Torres, P., \& Madera, C. (2008). Reuso de aguas residuales domésticas en agricultura. Una revisión. Agronomía Colombiana. https://www.redalyc.org/pdf/1803/180314732020. pdf

- Simonete, M. A., Kiehl, J. de C., Andrade, C. A., \& Teixeira, C. F. A. (2003). Efeito do lodo de esgoto em um Argissolo e no crescimento e nutrição de milho. Pesquisa Agropecuária Brasileira, 38(10), 1187-1195. https://doi.org/10.1590/s0100204x2003001000008

- Singh, S., Singh, J., \& Pal Vig, A. (2016). Earthworm as ecological engineers to change the physico-chemical properties of soil: Soil vs vermicast. Ecological Engineering, 90,1-5. https://doi.org/10.1016/j.ecoleng.2016.01.072

- SINIA. (2012). Mapa de suelos en los distritos de Lima | SINIA | Sistema Nacional de Información Ambiental. MINAM. https://sinia.minam.gob.pe/mapas/mapa-suelos- distritos-lima

- Su, C., Zhang, F.,Cui, X., Cheng, Z., Zheng, Z. (2020). Source characterization of nitrate in groundwater using hydrogeochemical and multivariate statistical analysis in the MulingXingkai Plain, Northeast China. Environmental Monitoring and Assessment, 192(7):456. https://doi.org/10.1007/s10661-020-08347-6

- Toonen, W. H. J., Macklin, M. G., Dawkes, G., Durcan, J. A., Leman, M., Nikolayev, Y., \& Yegorov, A. (2021). A hydromorphic reevaluation of the forgotten river civilizations of Central Asia. Proceedings of the National Academy of Sciences of the United States of America, 117(52), 3298232988. https://doi.org/10.1073/PNAS.2009553117

- Valdes, R., Aguilera, G., Tobón, E., Samaniego, M., Díaz, J., \& Hernández, C. (2019). Potential Uses of Treated Municipal Wastewater in a Semiarid Region of Mexico. Sustainability, 11(8), 2217. https://doi.org/10.3390/su11082217

- World Economic Forum. (2020). The Global Risks Report 2020 Insight Report 15th Edition. In The Global Risks Report 2020. http://www3.weforum.org/docs/WEF_Global_Ris k_Report_2020.pdf

- Zamora, F., Rodríguez, N., Torres, D., \& Yendis, H. (2008). Efecto del riego con aguas residuales sobre propiedades químicas de suelos de la planicie de Coro, estado Falcón. Bioagro, 20, 193-199. https://www.redalyc.org/pdf/857/85714153006.pdf

- Zhang, W. Y., Wei, Z. W., Wang, B. H., \& Han, X. P. (2016). Measuring mixing patterns in complex networks by Spearman rank correlation coefficient. Physica A: Statistical Mechanics and Its Applications, $\quad 451, \quad 440-450$. https://doi.org/10.1016/j.physa.2016.01.056

- Zhang, Z., \& Furman, A. (2021). Soil redox dynamics under dynamic hydrologic regimes - A review. Science of The Total Environment, 763:143026. https://doi.org/10.1016/j.scitotenv.2020.143026

- Zhou, Y., \& Li, S. (2020). BP neural network modeling with sensitivity analysis on monotonicity based Spearman coefficient. Chemometrics and Intelligent Laboratory Systems, 200: 103977. https://doi.org/10.1016/j.chemolab.2020.103977 